Torsion sensor based on a figure-of-eight cavity fibre laser

This article has been downloaded from IOPscience. Please scroll down to see the full text article.

2013 Laser Phys. Lett. 10045105

(http://iopscience.iop.org/1612-202X/10/4/045105)

View the table of contents for this issue, or go to the journal homepage for more

Download details:

IP Address: 193.136.24.81

The article was downloaded on 27/02/2013 at $14: 13$

Please note that terms and conditions apply. 


\title{
LETTER
}

\section{Torsion sensor based on a figure-of-eight cavity fibre laser}

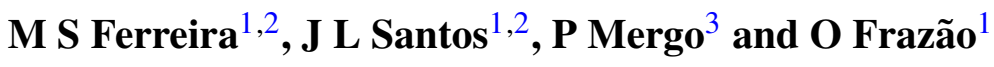 \\ ${ }^{1}$ INESC Porto, Rua do Campo Alegre, 687, 4169-007 Porto, Portugal \\ ${ }^{2}$ Faculdade de Ciências da Universidade do Porto, Rua do Campo Alegre, 687, 4169-007 Porto, Portugal \\ ${ }^{3}$ Laboratory of Optical Fibres Technology, Faculty of Chemistry, Maria Curie Sklodowska University, \\ Sklodowska Sq. 3, 20-031 Lublin, Poland \\ E-mail: msaf@inescporto.pt
}

Received 10 December 2012

Accepted for publication 17 December 2012

Published 27 February 2013

Online at stacks.iop.org/LPL/10/045105

\begin{abstract}
A figure-of-eight based fibre laser cavity is proposed for the measurement of torsion. In one of the loops a section of photonic crystal fibre is inserted, acting both as an optical filter and the sensing head. The laser emission depends on the optical filter polarization and length. A single lasing band is achieved with a full width at half maximum of $\sim 1.72 \mathrm{~nm}$. The proposed sensor presents sensitivity to torsion of $7.13 \mathrm{pm} /$ degree over a range of $180^{\circ}$. This configuration can also be used to measure optical power variations. Besides, due to the properties of the optical filter this sensor presents low sensitivity to temperature.
\end{abstract}

(Some figures may appear in colour only in the online journal)

\section{Introduction}

Ring-based laser cavities have been widely explored in the past. When compared to linear cavities, the unidirectional travelling wave obtained eliminates both the backscattering and the spatial-hole burn effects [1]. Thus, these cavities present good stability, flexibility and are easy to manufacture [2].

Optical fibre lasers with a figure-of-eight configuration have been reported for multi-wavelength emission using both erbium-doped fibre [3] and erbium-ytterbium co-doped fibre [4-6] as the gain medium. The inclusion of an optical filter in the loop can be decisive in achieving good output stability [7]. Zhou et al reported the insertion of a filter in a ring cavity to generate four-wavelength emission with equal frequency [1]. With this configuration, dual-wavelength lasing action was also attained. The use of a twin-core photonic crystal fibre (PCF) to create a Mach-Zehnder interferometer was reported by Kim et al to obtain a wavelength selective comb filter [8]. Tan et al observed that when a PCF section was introduced into the cavity, higher output and more lasing wavelengths could be achieved [9].
Furthermore, PCFs have been explored as passive torsion sensors, proving to be sensitive to this measurand and insensitive to temperature and strain [10]. Thus, when combining both sensing and lasing properties, high resolution, small loss and low cost systems can be achieved [10,11].

In the context of active sensors, Shi et al presented a Mach-Zehnder interferometer formed by a pair of long period gratings inserted in a ring laser to perform active torsion measurements [12]. Wei et al [13] proposed a photonic crystal fibre-based fibre ring laser with high torsion sensitivity for a small angular range.

In this letter, a laser cavity based on the figure-of-eight configuration is presented to perform torsion measurements. A section of commercial PCF is inserted into one of the loops, acting both as an optical filter and as the sensing head. The torsion will induce a modification in the sensing head cores, and it can be applied over a range of $180^{\circ}$.

\section{Experimental results}

In a first stage of this work, the interferometric filter was designed to provide the proper characteristics to be used in 


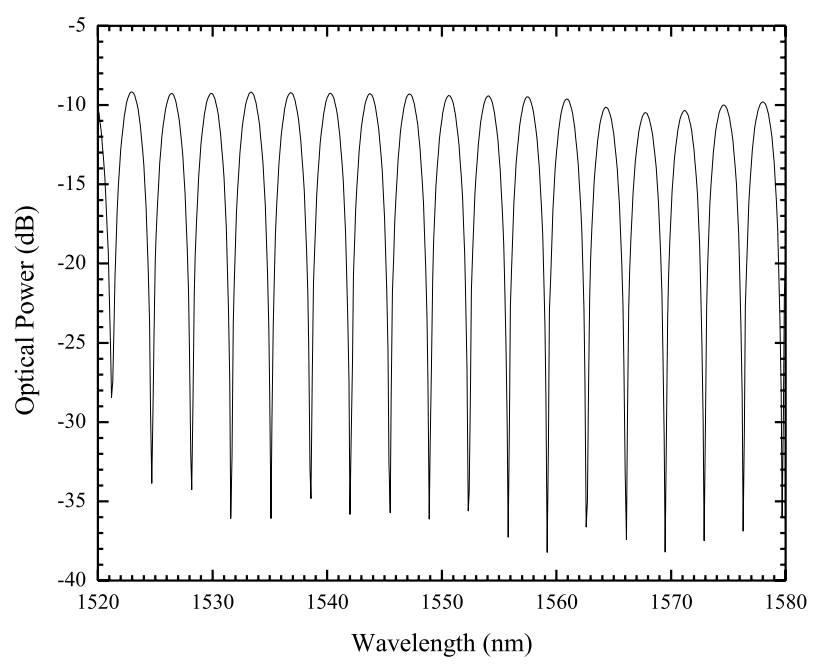

Figure 1. Optical spectrum of the interferometric filter.

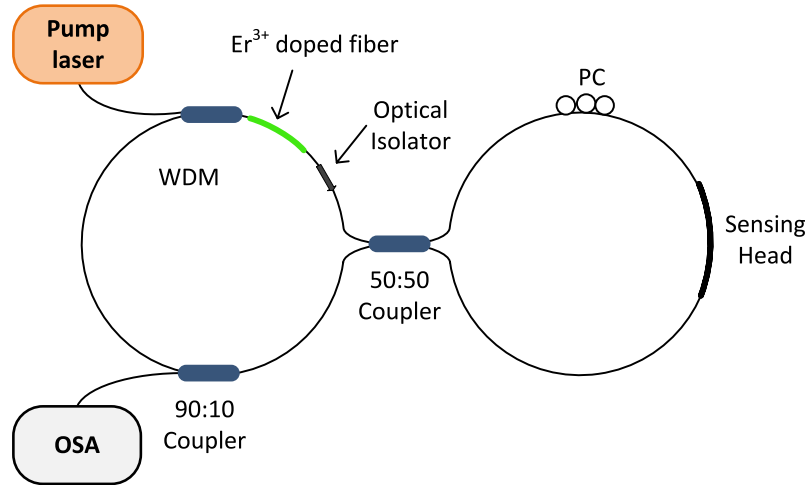

Figure 2. Scheme of the experimental setup.

Table 1. Properties of the interferometric filter (FWHM, full width at half maximum).

\begin{tabular}{ll}
\hline & Average value \\
\hline FWHM (nm) & 1.72 \\
Channel spacing (THz) & 0.43 \\
Channel pass-band (THz) & 0.17 \\
\hline
\end{tabular}

the figure-of-eight configuration. The filter was made up of $78.6 \mathrm{~cm}$ long polarization maintaining (PM) PCF section. The PM PCF used in this experiment was a commercial one (PM-1550-01 from Thorlabs). The channelled spectral behaviour can be seen in figure 1 . The main properties of this interferometric filter are gathered in table 1.

The experimental setup, presented in figure 2, had a figure-of-eight configuration. One of the loops was made up of a pump diode laser emitting at $980 \mathrm{~nm}$, a 980/1550 nm wavelength division multiplexer (WDM), an optical isolator, a 90:10 optical coupler and an optical spectrum analyser (OSA) to perform the readings. In between the WDM and the optical isolator, a section of erbium-doped fibre (about $80 \mathrm{~cm}$ long) was inserted to provide the gain. The concentrations of the erbium and aluminum ions were $1000 \mathrm{ppm}$ and $10000 \mathrm{ppm}$, respectively. The numerical aperture of the erbium-doped fibre was 0.27 , its core diameter was $\sim 5 \mu \mathrm{m}$ and it presented a

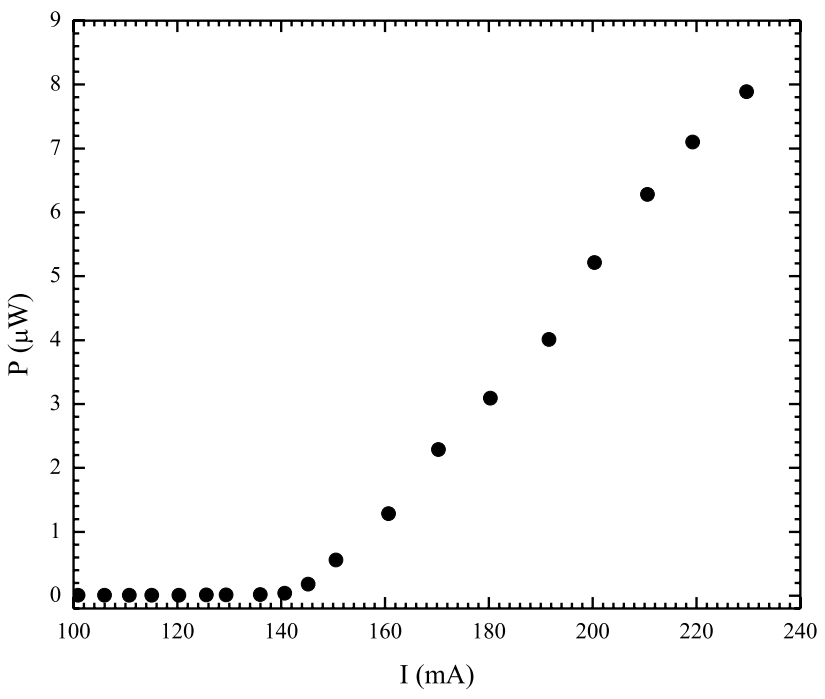

Figure 3. Optical power variations with drive-in current.

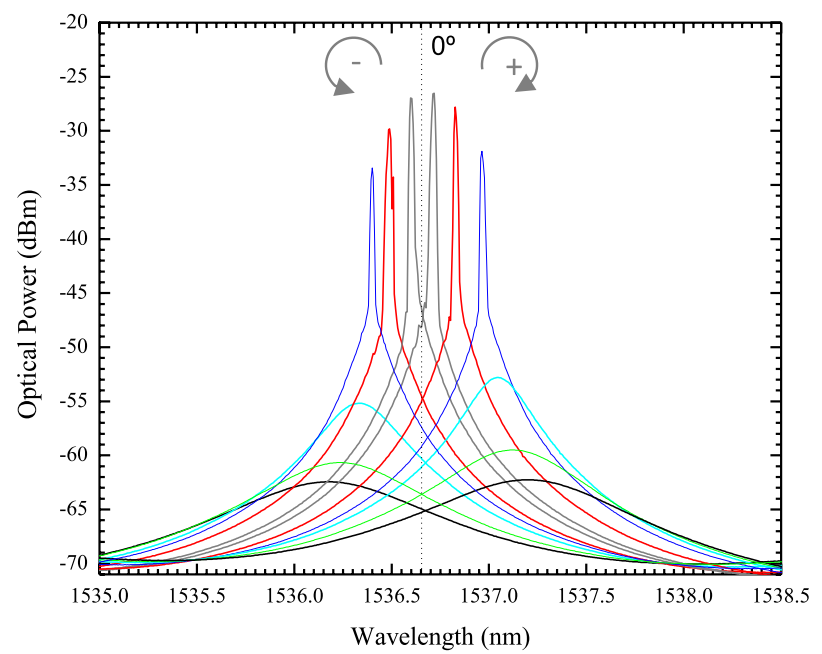

Figure 4. Variation of the laser emission with the applied torsion.

step index profile. The other loop had a polarization controller (PC) and the interferometric filter previously described, which was used as a sensing head. Depending on the polarization of light travelling inside this loop, one or more lasing peaks were obtained. Since this configuration was used to measure torsion variations, one single peak laser was considered throughout the experiment. In between the two loops there was a $3 \mathrm{~dB}$ optical coupler.

The response of the laser power to the diode drive-in current is shown in figure 3. The current threshold was estimated to be $145.5 \mathrm{~mA}$ and a maximum power of $\sim 7.9 \mu \mathrm{W}$ was achieved for a drive-in current of $\sim 230 \mathrm{~mA}$.

In order to perform torsion measurements, one side of the sensing head was introduced in a torsion stage, with a resolution of $0.5^{\circ}$, while the other was kept fixed. It was found that both the amplitude and central wavelength of the laser peak shifted as torsion was applied, both for positive and negative angles. This behaviour, which can be observed in figure 4 , occurs due to rotation of the PM PCF cores. Starting from $0^{\circ}$, as the angles increased the peak shifted towards 


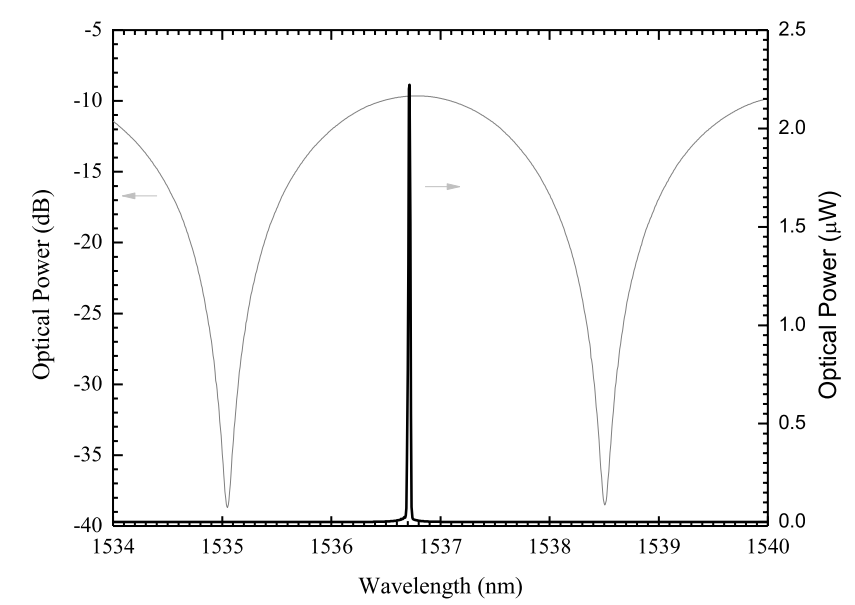

Figure 5. Interferometric filter spectrum (grey line) and laser spectrum (black line).

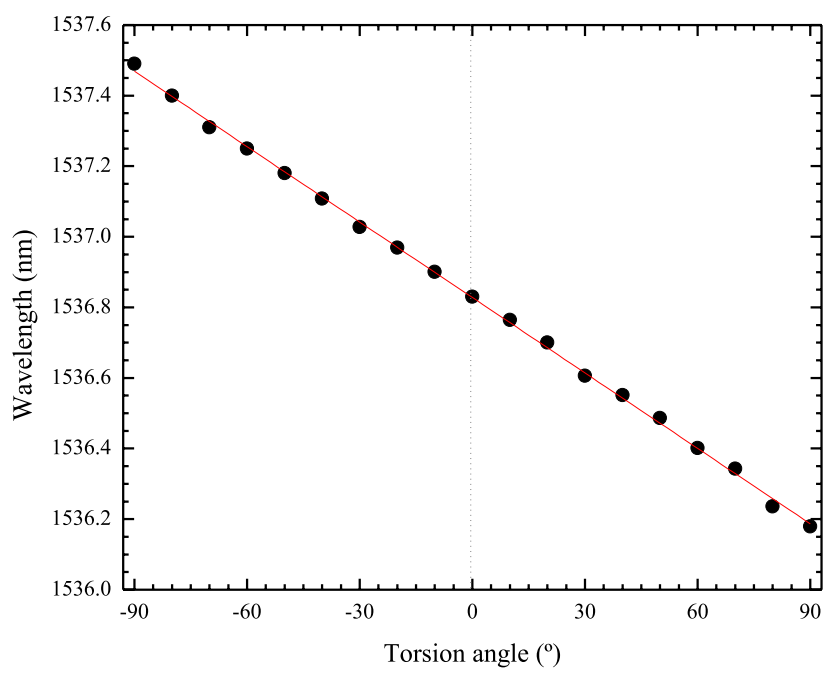

Figure 6. Wavelength variation with applied torsion.

higher wavelengths (red shift). Accordingly, by decreasing the angle from $0^{\circ}$ to $-90^{\circ}$, a shift towards lower wavelengths (blue shift) was observed. The applied torsion range was $\left[-90^{\circ} ; 90^{\circ}\right]$. However, the behaviour is periodic, according to the filter spectrum (see figure 5).

Considering the variation of the wavelength with the applied torsion (see figure 6), there is a linear decrease in wavelength for this torsion angle range, which led to sensitivity of $-7.13 \mathrm{pm} /$ degree. It should be noticed that the analysis could also be applied to variations of optical power, since the laser emission varies depending where it is placed in relation to the interferometric filter. Thus, when the wavelength corresponds to a maximum at the interferometric filter spectrum, a maximum also occurs in the laser peak emission, decreasing according to the behaviour of the filter.

\section{Conclusions}

In summary, a torsion active sensor based on a figure-of-eight laser cavity was demonstrated. The interferometric filter was made up of a section of photonic crystal fibre which also acted as a sensing element. In order to achieve single band laser emission, a polarization controller was inserted into one of the loops. Torsion was applied over a range of $180^{\circ}$ and both wavelength and optical power changed, the latter being due to the properties of the optical filter. A sensitivity of $-7.13 \mathrm{pm} /$ degree was obtained. Since the filter presents low sensitivity to temperature, in addition to its low cost, ease of fabrication and reliable results, it proves to be a good choice for performing active torsion measurements.

\section{References}

[1] Zhou K J and Ruan Y F 2010 Fiber ring laser employing an all-polarization-maintaining loop periodic filter Laser Phys. 20 1449-52

[2] Sun G, Chung Y and Moon D S 2008 L-band tunable multiwavelength fiber laser using an unpumped polarization maintaining an erbium-ytterbium double-clad fiber-loop mirror Laser Phys. 18 1196-9

[3] Zhang Z X, Xu K, Wu J, Hong X B and Lin J T 2008 Multiwavelength figure-of-eight fiber laser with a nonlinear optical loop mirror Laser Phys. Lett. 5 213-6

[4] Ye W, Liu W, Chen T, Yang D Z and Shen Y H 2010 Erbium-ytterbium co-doped multi-wavelength double-clad fiber laser around $1612 \mathrm{~nm}$ Laser Phys. 20 1636-40

[5] Ahmad B A, Al-Alimi A W, Abas A F, Harun S W and Mahdi M A 2012 Stable double spacing multiwavelength Brillouin-erbium doped fiber laser based on highly nonlinear fiber Laser Phys. 22 977-81

[6] Ye W, Wang J, Chen T and Shen Y H 2011 Multi-wavelength mode-locked $\mathrm{Er} / \mathrm{Yb}$ co-doped fiber laser with square nano-second pulse output Laser Phys. 21 1784-8

[7] Liang P S, Zhang Z X, Kuang Q Q and Sang M H 2009 All-fiber birefringent filter with fine tunability and changeable spacing Laser Phys. 19 2124-8

[8] Kim B K and Chung Y 2012 Tunable and switchable SOA-based multi-wavelength fiber laser using twin-core photonic crystal fiber Laser Phys. Lett. $9734-8$

[9] Tan S J, Harun S W, Shahabuddin N S, Arof H and Ahmad H 2012 Dual-cavity dual-output multi-wavelength fiber laser based on nonlinear polarization effect Laser Phys. 22 1601-5

[10] Fu H Y, Khijwania S K, Tam H Y, Wai P K A and Lu C 2010 Polarization-maintaining photonic-crystal-fiber-based all-optical polarimetric torsion sensor Appl. Opt. 49 5954-8

[11] Nalawade S M, Harnol S S and Thakur H V 2012 Temperature and strain independent modal interferometric torsion sensor using photonic crystal fiber IEEE Sens. J. 12 2614-5

[12] Shi L, Zhu T, Fan Y, Chiang K S and Rao Y 2011 Torsion sensing with a fiber ring laser incorporating a pair of a rotary long-period fiber gratings Opt. Commun. 284 5299-302

[13] Wei S, Zhang W, Shang J and Yin L 2012 Torsion sensing characteristics of fibre ring laser based on nonlinear polarization rotation Electron. Lett. 48 116-8 